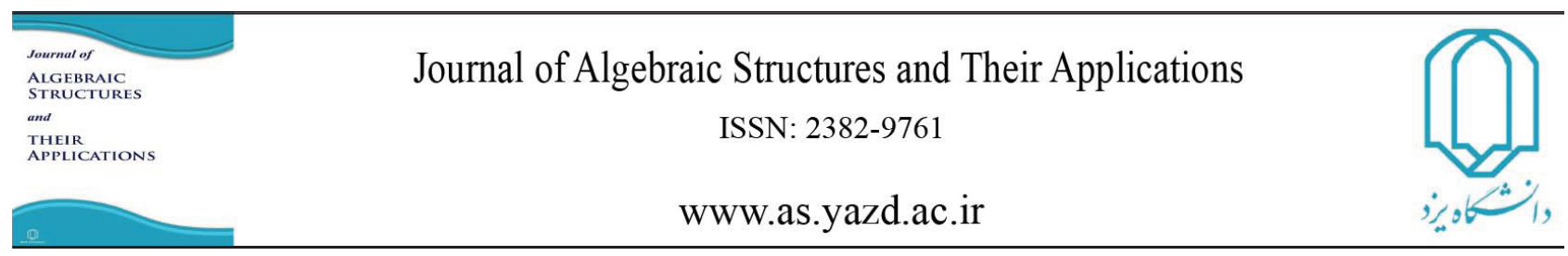

Algebraic Structures and Their Applications Vol. 5 No. 2 ( 2018 ) pp 45-53.

\title{
NO-HOMOMORPHISM CONDITIONS FOR HYPERGRAPHS
}

\author{
SAMANEH TAHMASEBI AND SADEGH RAHIMI SHERBAF
}

Communicated by M.A. Iranmanesh

\begin{abstract}
In this paper, we define some new homomorphism-monotone parameters for hypergraphs. Using these parameters, we extend some graph homomorphism results to hypergraph case. Also, we present some bounds for some well-known invariants of hypergraphs such as fractional chromatic number,independent numer and some other invariants of hyergraphs, in terms of these parameters.
\end{abstract}

\section{INTRODUCTION}

Graph homomorphism is known as a fundamental problem in graph theory, since many problems in this area can be interpreted by the existence of homomorphism between some related graphs, for instance, see [5, $6, \mathbf{8}, \mathbf{8}, \mathbf{9}, \mathbf{1 0}, \mathbf{1}]$. As expected, on the other hand, graph homomorphism is an NP-hard problem, which as a matter of fact, emphasizes that finding some necessary conditions for the existing of graph homomorphism are valuable. This problem has been received a considerable attention in the literature, see [2, 3, [7, ए2].

DOI:http://dx.doi.org/10.29252/asta.5.2.45

MSC(2010): Primary:05C50

Keywords: hypergraph homomorphism, independing number, clique number, chromatic number, fractional chromatic number.

Received: 09 May 2018, Accepted: 01 Jan 2019

*Corresponding author: samanehtahmasebi@shahroodut.ac.ir 
In this paper, we will lift the problem of the existence of graph homomorphism to the case of hypergraphs. We generalize some known results $[2,4,7]$ to hypergraph case. To state our results, we first need to set up some notation and terminology.

Notations: Hereafter, two symbols $[m]$ and $\left(\begin{array}{c}{[m]} \\ n\end{array}\right)$ stand respectively for the set $\{1,2, \ldots, m\}$ and the collection of all $n$-subsets of $[m]$. A hypergraph $\mathcal{H}$ is a pair $(V, E)$, where $V$ is a finite set of point called vertices, and $E$ is a set of subsets of $V$, called edges. A hypergraph is called $r$-uniform if all of its edges have the same cardinality $r$. A hypergraph $\mathcal{H}^{\prime}=\left(V\left(\mathcal{H}^{\prime}\right), E\left(\mathcal{H}^{\prime}\right)\right)$ is called a subhypergraph of a hypergraph $\mathcal{H}=(V(\mathcal{H}), E(\mathcal{H}))$ if $V\left(\mathcal{H}^{\prime}\right) \subseteq V(\mathcal{H})$ and $E\left(\mathcal{H}^{\prime}\right) \subseteq E(\mathcal{H})$. An induced subhypergraph of a hypergraph $\mathcal{H}$ is a subhypergraph which is given by a subset of the vertices of $\mathcal{H}$, and contains the same edges regarding these vertices as in the original hypergraph. A clique of an $r$-uniform hypergraph $\mathcal{H}$ is a subset of its vertices such that every $r$ vertices in this subset form an edge in $\mathcal{H}$. The size of the largest clique in $\mathcal{H}$ is called the clique number of $\mathcal{H}$ and denoted by $\omega(\mathcal{H})$. A set $S \subseteq V(\mathcal{H})$ is called independent if there is no edge $e \in E(\mathcal{H})$ contained in $S$. The size of the largest independent set is called independent number and denoted by $\alpha(\mathcal{H})$. For two hypergraphs $\mathcal{G}$ and $\mathcal{H}$, a homomorphism from $\mathcal{G}$ to $\mathcal{H}$ is a mapping $f: V(\mathcal{G}) \longrightarrow V(\mathcal{H})$ such that for every edge $e \in E(\mathcal{G})$, there exists at least one edge $e^{\prime} \in E(\mathcal{H})$ such that $e^{\prime} \subseteq f(e)$. We write $\mathcal{G} \longrightarrow \mathcal{H}($ resp. $\mathcal{G} \nrightarrow \mathcal{H}$ ) if there exists a homomorphism ( resp. no-homomorphism) from $\mathcal{G}$ to $\mathcal{H}$. If a homomorphism is bijective then it is called an isomorphism. Note that, the clique number of an $r$-uniform hypergraph $\mathcal{H}$ is the maximum integer $n$ for which $K_{n}^{r} \longrightarrow \mathcal{H}$, where $K_{n}^{r}$ is an $r$-uniform hypergraph with vertex set $[n]$ and edge set consisting of all $r$-subsets of $[n]$. For $r=2$, we omit the super index and write $K_{n}$ instead of $K_{n}^{2}$. An isomorphism from a hypergraph $\mathcal{G}$ to itself is called an automorphism. A hypergraph $\mathcal{H}$ is called vertex transitive if for every vertices $x, y \in V(\mathcal{H})$, there is an automorphism $f: \mathcal{H} \longrightarrow \mathcal{H}$ such that $f(x)=y$. A hypergraph $\mathcal{H}$ is called edge transitive if for every edges $e_{1}, e_{2} \in E(\mathcal{H})$, there is an automorphism $f: \mathcal{H} \longrightarrow \mathcal{H}$ such that $f\left(e_{1}\right)=e_{2}$. Let $m \geq 2 n$ be positive integers. The Kneser graph $\operatorname{KG}(m, n)$ has vertex set $\left(\begin{array}{c}{[m]} \\ n\end{array}\right)$, and each pair of vertices make an edge, if their intersection is empty.

A proper coloring of a hypergraph is an assignment of colors (natural numbers) to the vertices of the hypergraph so that every edge receive at least two different colors. A $t$-coloring of a hypergraph $\mathcal{H}$ is a coloring of $\mathcal{H}$ using at most $t$ colors. For a hypergraph $\mathcal{H}$ its chromatic number $\chi(\mathcal{H})$ is the smallest integer $t$ for which $\mathcal{H}$ admits a $t$-coloring. Note that the chromatic number of a hypergraph $\mathcal{H}$ is the minimum integer $n$ for which $\mathcal{H} \longrightarrow K_{n}$. Also, the fractional chromatic number of a hypergraph $\mathcal{H}$, denoted by $\chi_{f}(\mathcal{H})$, is defined as follows,

$$
\chi_{f}(\mathcal{H})=\inf \left\{\frac{m}{n} \mid \mathcal{H} \longrightarrow \operatorname{KG}(m, n)\right\}
$$


It is known that the infimum can be replaced by minimum. Also, since $\operatorname{KG}(m, 1)=K_{m}$, for any hypergraph $\mathcal{H}$, we clearly have $\chi_{f}(\mathcal{H}) \leq \chi(\mathcal{H})$.

\section{Conditions for existence of hypergraph homomorphism}

Let $\mathcal{H}$ be a hypergraph and $c$ be a proper coloring of $\mathcal{H}$. We define the vertex-sum coloring of $\mathcal{H}$ related to the coloring $c$ by $\sum_{c}(\mathcal{H})=\sum_{v \in V(\mathcal{H})} c(v)$. The vertex-chromatic sum of $\mathcal{H}$, denoted by $\sum(\mathcal{H})$, is defined as follows,

$$
\sum(\mathcal{H})=\min \left\{\sum_{c}(\mathcal{H}) \mid c \text { is a proper coloring of } \mathcal{H}\right\}
$$

The following theorem provides a necessary condition for the existence of hypergraph homomorphism extending a theorem in [2] to hypergraph case. In fact, we provide a necessary condition for the existence of hypergraph homomorphism by using the vertex-chromatic sum of a hypergraph.

Theorem 2.1. Let $\mathcal{G}$ and $\mathcal{H}$ be two hypergraphs such that $\mathcal{H}$ is a vertex transitive hypergraph. If $f: \mathcal{G} \longrightarrow \mathcal{H}$ is a homomorphism, then

$$
\frac{\sum(\mathcal{G})}{|V(\mathcal{G})|} \leq \frac{\sum(\mathcal{H})}{|V(\mathcal{H})|}
$$

Proof. Let $\widetilde{\mathcal{G}}=\bigcup_{\sigma \in A u t(\mathcal{H})} \mathcal{G}_{\sigma}$, where each $\mathcal{G}_{\sigma}$ is a copy of $\mathcal{G}$. Define $g: \widetilde{\mathcal{G}} \longrightarrow \mathcal{H}$ such that $g(v)=\sigma \circ f(v)$ whenever $v$ is a vertex of $\mathcal{G}_{\sigma}$. Obviously, $g$ is a homomorphism and since $\mathcal{H}$ is a vertex transitive hypergraph, one can easily check that for each vertex $x \in V(\mathcal{H}),\left|g^{-1}(x)\right|$ is a constant, i.e., independent of $x$. To see this, note that for any two vertices $x, y \in V(\mathcal{H})$, there is an automorphism $\sigma \in A u t(\mathcal{H})$ such that $\sigma(x)=y$. Now, it is clear that $\sigma \circ g$ is same as $g$ with respect to a renaming of the copies of $\mathcal{G}$ in $\widetilde{\mathcal{G}}$ which implies that $\left|g^{-1}(x)\right|=$ $\left|(\sigma \circ g)^{-1}(x)\right|$. On the other hand, since $\sigma(x)=y$, we have $\left|g^{-1}(x)\right|=\left|(\sigma \circ g)^{-1}(y)\right|$ resulting in $\left|g^{-1}(x)\right|=\left|g^{-1}(y)\right|$. For simplicity of notation, set $\mathfrak{Z}=\left|g^{-1}(x)\right|$. Consequently, $\left|g^{-1}(V(\mathcal{H}))\right|=$ $\sum_{x \in V(\mathcal{H})}\left|g^{-1}(x)\right|=\mathfrak{Z} \cdot|V(\mathcal{H})|$. On the other hand, $\left|g^{-1}(V(\mathcal{H}))\right|=|A u t(\mathcal{H})| \cdot|V(\mathcal{G})|$, which implies $\mathfrak{Z}=\frac{|\operatorname{Aut}(\mathcal{H})| \cdot|V(\mathcal{G})|}{|V(\mathcal{H})|}$. Let $c$ be a proper coloring of $\mathcal{H}$ such that $\sum_{c}(\mathcal{H})=\sum(\mathcal{H})$. For each $v \in V(\widetilde{G})$, set $\widetilde{c}(v)=c(g(v))$. This is a proper coloring of $\widetilde{G}$ and $\sum_{\widetilde{c}}(\widetilde{G})=\frac{|A u t(\mathcal{H})| \cdot|V(\mathcal{G})|}{|V(\mathcal{H})|} \times \sum(\mathcal{H})$. Therefore, there is an $\sigma$ such that $\sum_{\widetilde{\mathcal{C}}_{\mathcal{G}_{\sigma}}}\left(\mathcal{G}_{\sigma}\right) \leq \frac{|V(\mathcal{G})|}{|V(\mathcal{H})|} \times \sum(\mathcal{H})$ and since $\mathcal{G}_{\sigma}$ is isomorphic to $\mathcal{G}$, we have $\sum(\mathcal{G}) \leq \frac{|V(\mathcal{G})|}{|V(\mathcal{H})|} \times \sum(\mathcal{H})$ which is the desired conclusion.

Let $\mathcal{H}$ be a hypergraph with $\chi(\mathcal{H})=n$. Since there is a homomorphism from $\mathcal{H}$ to $K_{n}$, Theorem [2.] implies

$$
\frac{\sum(\mathcal{H})}{|V(\mathcal{H})|} \leq \frac{\sum\left(K_{n}\right)}{\left|V\left(K_{n}\right)\right|}=\frac{n(n+1)}{2 n}=\frac{n+1}{2}=\frac{\chi(\mathcal{H})+1}{2}
$$


and thus

$$
\sum(\mathcal{H}) \leq \frac{\chi(\mathcal{H})+1}{2} \times|V(\mathcal{H})| .
$$

For two hypergraphs $\mathcal{H}$ and $\mathcal{K}$ define

$$
\alpha_{\mathcal{K}}(\mathcal{H})=\max \{|S| \mid S \subseteq V(\mathcal{H}), \mathcal{H}[S] \longrightarrow \mathcal{K}\}
$$

i.e., $\alpha_{\mathcal{K}}(\mathcal{H})$ is the maximum number of vertices of hypergraph $\mathcal{H}$ which the induced subhypergraph by these vertices is homomorphic to $\mathcal{K}$. By the following theorem, we generalize a graph homomorphism result by Bondy and Hell [4] to the hypergraph case.

Theorem 2.2. Let $\mathcal{G}$ and $\mathcal{H}$ be two hypergraphs and $\mathcal{H}$ be a vertex transitive hypergraph. For each hypergraph $\mathcal{K}$, if $f: \mathcal{G} \longrightarrow \mathcal{H}$ is a homomorphism, then

$$
\frac{\alpha_{\mathcal{K}}(\mathcal{H})}{|V(\mathcal{H})|} \leq \frac{\alpha_{\mathcal{K}}(\mathcal{G})}{|V(\mathcal{G})|}
$$

Proof. Let $\widetilde{\mathcal{G}}=\bigcup_{\sigma \in A u t(\mathcal{H})} \mathcal{G}_{\sigma}$, where each $\mathcal{G}_{\sigma}$ is a copy of $\mathcal{G}$. Define $g: \widetilde{\mathcal{G}} \longrightarrow \mathcal{H}$ such that $g(v)=\sigma \circ f(v)$. Obviously, $g$ is a homomorphism and we have

$$
\alpha_{\mathcal{K}}(\widetilde{\mathcal{G}})=|A u t(\mathcal{H})| \times \alpha_{\mathcal{K}}(\mathcal{G})
$$

Consider $S \subseteq V(\mathcal{H})$ such that $\mathcal{H}[S] \longrightarrow \mathcal{K}$ and $|S|=\alpha_{\mathcal{K}}(\mathcal{H})$. For $S^{\prime}=g^{-1}(S) \subseteq V(\widetilde{\mathcal{G}})$, we have $\widetilde{\mathcal{G}}\left[S^{\prime}\right] \longrightarrow \mathcal{K}$ since $\widetilde{\mathcal{G}}\left[S^{\prime}\right] \longrightarrow \mathcal{H}[S] \longrightarrow \mathcal{K}$. So $\left|S^{\prime}\right| \leq \alpha_{\mathcal{K}}(\widetilde{\mathcal{G}})$. On the other hand, we have $\left|S^{\prime}\right|=$ $\sum_{x \in S}\left|g^{-1}(x)\right|$. With the same proof as in Theorem 2.1 , we have $\left|g^{-1}(x)\right|=|A u t(\mathcal{H})||V(\mathcal{G})|$ for each $x \in V(\mathcal{H})$. Consequently, we have

$$
\left|S^{\prime}\right|=\sum_{x \in S}\left|g^{-1}(x)\right|=\left|g^{-1}(x)\right| \cdot|S| \leq \alpha_{\mathcal{K}}(\widetilde{\mathcal{G}})=|A u t(\mathcal{H})| \times \alpha_{\mathcal{K}}(\mathcal{G}),
$$

Thus, $|A u t(\mathcal{H})| \frac{|V(\mathcal{G})|}{|V(\mathcal{H})|} \cdot \alpha_{\mathcal{K}}(\mathcal{H}) \leq|A u t(\mathcal{H})| \cdot \alpha_{\mathcal{K}}(\mathcal{G})$, and $\frac{\alpha_{\mathcal{K}}(\mathcal{H})}{|V(\mathcal{H})|} \leq \frac{\alpha_{\mathcal{K}}(\mathcal{G})}{|V(\mathcal{G})|}$, as desired

For a hypergraph $\mathcal{H}$, we remind that a subset of $V(\mathcal{H})$ such that the induced subhypergraph by them has no edge is an independent set of $\mathcal{H}$. The maximum cardinality for such a subset is called the independence number of $\mathcal{H}$ and denoted by $\alpha(\mathcal{H})$. It is clear that if there is a homomorphism from $\mathcal{H}$ to $K_{1}$, then $\mathcal{H}$ must have no edge and vice versa. Consequently, for each hypergraph $\mathcal{H}$, we have $\alpha_{K_{1}}(\mathcal{H})=\alpha(\mathcal{H})$, because $\alpha_{K_{1}}(\mathcal{H})=\max \{|S|: S \subseteq V(\mathcal{H}), \mathcal{H}[S] \longrightarrow$ $K_{1}$ \}, which results in the well-known inequality $\chi(\mathcal{H}) \geq \frac{|V(\mathcal{H})|}{\alpha(\mathcal{H})}$. To see this, note that if $\chi(\mathcal{H})=n$, then there is a homomorphism from $\mathcal{H}$ to $K_{n}$, and accordingly by Theorem 2.2 , $\frac{\alpha(\mathcal{H})}{|V(\mathcal{H})|} \geq \frac{\alpha_{K_{1}}\left(K_{n}\right)}{\left|V\left(K_{n}\right)\right|}=\frac{1}{n}$. As applications of Theorem [2.2, we have the next tow corollaries as well.

Corollary 2.3. For any vertex transitive $r$-uniform hypergraph $\mathcal{H}$, we have $\omega(\mathcal{H}) \cdot \alpha(\mathcal{H}) \leq$ $(r-1)|V(\mathcal{H})|$. 
Proof. If $\omega(\mathcal{H})=n$, then there is a homomorphism from $K_{n}^{r}$ to $\mathcal{H}$. Now, by Theorem ए2.2, we have $\frac{\alpha_{K_{1}}(\mathcal{H})}{|V(\mathcal{H})|} \leq \frac{\alpha_{K_{1}}\left(K_{n}^{r}\right)}{\left|V\left(K_{n}^{r}\right)\right|}=\frac{r-1}{n}$, which finishes the proof.

Corollary 2.4. For any hypergraph $\mathcal{H}$, the size of the largest bipartite subhypergraph of $\mathcal{H}$ is more than $2\left(1-\frac{1}{\chi_{f}(\mathcal{H})}\right) \frac{|V(\mathcal{H})|}{\chi_{f}(\mathcal{H})}$.

Proof. Let $\chi_{f}(\mathcal{H})=\frac{m}{n}$, then there is a homomorphism from $\mathcal{H}$ to $\mathrm{KG}(t m, t n)$ for some positive integer $t$. Now, by Theorem [2.2, we have

$$
\frac{\alpha_{K_{2}}(\mathrm{KG}(t m, t n))}{|V(\mathrm{KG}(t m, t n))|} \leq \frac{\alpha_{K_{2}}(\mathcal{H})}{|V(\mathcal{H})|}
$$

Define

$$
\begin{aligned}
& A=\{X \subseteq[t m]|| X \mid=t n, 1 \in X, 2 \notin X\} \\
& B=\{X \subseteq[t m]|| X \mid=t n, 2 \in X, 1 \notin X\} .
\end{aligned}
$$

Let $K_{0}$ be the induced subgraph of $\mathrm{KG}(t m, t n)$ by the vertices in $A \cup B$. It is simple to see that $K_{0}$ is bipartite and thus

$$
\left.2\left(\begin{array}{c}
t m-2 \\
t n-1
\end{array}\right)=\left|V\left(K_{0}\right)\right| \leq \alpha_{K_{2}}(\mathrm{KG}(t m, t n))\right) .
$$

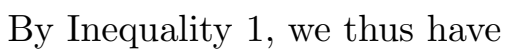

$$
\begin{aligned}
\frac{\alpha_{K_{2}}(\mathcal{H})}{|V(\mathcal{H})|} & \geq \frac{\alpha_{K_{2}}(\mathrm{KG}(t m, t n))}{|V(\mathrm{KG}(t m, t n))|} \\
& \geq \frac{\left|V\left(K_{0}\right)\right|}{|V(\mathrm{KG}(t m, t n))|} \\
& =\frac{2\left(\begin{array}{c}
t m-2 \\
t n-1
\end{array}\right)}{\left(\begin{array}{c}
t m \\
t n
\end{array}\right)} \\
& =\frac{2 n(t m-t n)}{m(t m-1)}>\frac{2 n(m-n)}{m^{2}} \\
& =2\left(1-\frac{1}{\chi_{f}(\mathcal{H})}\right) \frac{1}{\chi_{f}(\mathcal{H})} .
\end{aligned}
$$

It is known that for a graph $G, \chi_{f}(G) \geq \frac{|V(G)|}{\alpha(G)}$. In the following, using Theorem ए.2, we extend this result to the case of hypergraphs.

Observation: For any hypergraph $\mathcal{H}$, we have $\chi_{f}(\mathcal{H}) \geq \frac{|V(\mathcal{H})|}{\alpha(\mathcal{H})}$. 
Proof. Let $\chi_{f}(\mathcal{H})=\frac{m}{n}$, then there is a homomorphism from $\mathcal{H}$ to $\mathrm{KG}(t m, t n)$, for some positive integer $t$. Now by Theorem [2.2, we have

$$
\frac{\alpha(\mathcal{H})}{|V(\mathcal{H})|} \geq \frac{\alpha(\mathrm{KG}(t m, t n))}{|V(\mathrm{KG}(t m, t n))|}=\frac{\left(\begin{array}{c}
t m-1 \\
t n-1
\end{array}\right)}{\left(\begin{array}{c}
t m \\
t n
\end{array}\right)}=\frac{n}{m},
$$

as desired.

Free independent set of graphs was defined by Alishahi and Hajiabolhassan [3] as a tool for studying the circular chromatic number of Kneser graphs. It was generalized to hypergraphs in [I], where some results for circular chromatic number of Kneser hypergraphs was obtained as well. For a hypergraph $\mathcal{H}$, a set $S \subseteq V(\mathcal{H})$ is called free independent if there are two distinct maximal independent sets $S_{1}, S_{2} \subseteq V(\mathcal{H})$ such that $S \varsubsetneqq S_{1}$ and $S \varsubsetneqq S_{2}$. The size of the largest free independent set of $\mathcal{H}$ is denoted by $\bar{\alpha}(\mathcal{H})$. In what follows, we extend the definition of $\alpha_{\mathcal{K}}(\mathcal{H})$ to $\bar{\alpha}_{\mathcal{K}}(\mathcal{H})$ such that, as $\alpha_{\mathcal{K}}(\mathcal{H})$ is an extension of independent set, $\bar{\alpha}_{\mathcal{K}}(\mathcal{H})$ is an extension of free independent set, that is, $\alpha_{K_{1}}(\mathcal{H})=\alpha(\mathcal{H})$ and $\bar{\alpha}_{K_{1}}(\mathcal{H})=\bar{\alpha}(\mathcal{H})$. For two hypergraphs $\mathcal{H}$ and $\mathcal{K}$, a subset $S \subseteq V(\mathcal{H})$ is called free with respect to $\mathcal{K}$, if there is a homomorphism from $\mathcal{H}[S] \longrightarrow \mathcal{K}$ and furthermore, there are two distinct maximal sets $S_{1}, S_{2} \subseteq V(\mathcal{H})$ with respect to the existence of homomorphism from $\mathcal{H}\left[S_{i}\right]$ to $\mathcal{K}$ such that $S \varsubsetneqq S_{1}$ and $S \varsubsetneqq S_{2}$. Now, define

$$
\bar{\alpha}_{\mathcal{K}}(\mathcal{H})=\max \{|S|: S \subseteq V(\mathcal{H}) \text { and } S \text { is free with respect to } \mathcal{K}\} .
$$

If there is no such an $S$, we define $\bar{\alpha}_{\mathcal{K}}(\mathcal{H})=\infty$. Note that setting $\mathcal{K}=K_{1}$, we have $\bar{\alpha}_{K_{1}}(\mathcal{H})=$ $\bar{\alpha}(\mathcal{H})$. With a similar proof as one for Theorem [2.2, we have the following theorem.

Theorem 2.5. Let $\mathcal{G}$ and $\mathcal{H}$ be two hypergraphs where $\mathcal{H}$ is vertex transitive. If $f: \mathcal{G} \longrightarrow \mathcal{H}$ is an edge-onto homomorphism, then

$$
\frac{\bar{\alpha}_{\mathcal{K}}(\mathcal{H})}{|V(\mathcal{H})|} \leq \frac{\bar{\alpha}_{\mathcal{K}}(\mathcal{G})}{|V(\mathcal{G})|}
$$

Density of a hypergraph $\mathcal{H}$, denoted by $\xi(\mathcal{H})$, is defined as follows:

$$
\xi(\mathcal{H})=\max \{\bar{e}(\mathcal{G}): \mathcal{G} \leq \mathcal{H}\}
$$

where

$$
\bar{e}(\mathcal{G})=\frac{|E(\mathcal{G})|}{|V(\mathcal{G})|}
$$

and $\mathcal{G} \leq \mathcal{H}$ means $\mathcal{G}$ is an induced subhypergraph of $\mathcal{H}$. Note that when $\mathcal{G}$ is a graph (i.e., a 2-uniform hypergraph) $2 \bar{e}(\mathcal{G})$ is equal to the average degree of $\mathcal{G}$.

Theorem 2.6. Let $\mathcal{G}$ and $\mathcal{H}$ be two hypergraphs and $\mathcal{H}$ be an $r$-uniform vertex and edge transitive hypergraph. If $f: \mathcal{G} \longrightarrow \mathcal{H}$ is a homomorphism, then

$$
\frac{\xi(\mathcal{H})}{\xi(\mathcal{G})} \leq \frac{\bar{e}(\mathcal{H})}{\bar{e}(\mathcal{G})}
$$


Proof. Let $\widetilde{\mathcal{G}}$ be a hypergraph made by disjoint union of $|A u t(\mathcal{H})| \operatorname{copies}\left\{\mathcal{G}_{\sigma}\right\}_{\sigma \in A u t(\mathcal{H})}$ of $\mathcal{G}$. We define $g: \widetilde{\mathcal{G}} \longrightarrow \mathcal{H}$ such that $g$ on each copy $\mathcal{G}_{\sigma}$ is defined by $\sigma \circ f$. Obviously, $g$ is an onto (edge and vertex) homomorphism and for each $v \in V(\mathcal{H})$, we have

$$
\left|g^{-1}(v)\right|=\frac{|V(\mathcal{G})| \times|A u t(\mathcal{H})|}{|V(\mathcal{H})|} .
$$

This clearly implies

$$
\left|V\left(\mathcal{G}^{\prime}\right)\right|=\frac{|V(\mathcal{G})| \times|\operatorname{Aut}(\mathcal{H})|}{|V(\mathcal{H})|} \times\left|V\left(\mathcal{H}^{\prime}\right)\right|,
$$

where $\mathcal{H}^{\prime}$ is a subhypergraph of $\mathcal{H}$ for which

$$
\xi(\mathcal{H})=\bar{e}\left(\mathcal{H}^{\prime}\right)=\frac{\left|E\left(\mathcal{H}^{\prime}\right)\right|}{\left|V\left(\mathcal{H}^{\prime}\right)\right|}
$$

and $\mathcal{G}^{\prime}$ is the subhypergraph of $\widetilde{\mathcal{G}}$ induced by the vertices in $g^{-1}\left(V\left(\mathcal{H}^{\prime}\right)\right)$. Now, for an edge $e \in E(\mathcal{H})$, set

$$
g^{-1}(e)=\left\{e^{\prime} \in E(\widetilde{\mathcal{G}}): e \subseteq g\left(e^{\prime}\right)\right\} .
$$

Since $\mathcal{H}$ is edge transitive, $\left|g^{-1}(e)\right|$ is independent from $e$. To see this, note that for any two edges $e, e^{\prime} \in E(\mathcal{H})$, there is an automorphism $\sigma \in A u t(\mathcal{H})$ such that $\sigma(e)=e^{\prime}$. Now, it is clear that $\sigma \circ g$ is same as $g$ with respect to a renaming of the copies of $\mathcal{G}$ in $\widetilde{\mathcal{G}}$ which implies that $\left|g^{-1}(e)\right|=\left|(\sigma \circ g)^{-1}\left(e^{\prime}\right)\right|$. On the other hand, since $\sigma(e)=e^{\prime}$, we have $\left|g^{-1}(e)\right|=\left|(\sigma \circ g)^{-1}\left(e^{\prime}\right)\right|$ resulting in $\left|g^{-1}(e)\right|=\left|g^{-1}\left(e^{\prime}\right)\right|$. Now, let $\zeta=\left|g^{-1}(e)\right|$. Also, each edge $e^{\prime} \in E(\widetilde{\mathcal{G}})$ appears in a constant number of $g^{-1}(e)$ for different $e \in E(\mathcal{H})$. Let this number be $\gamma$. One can simply check that

$$
\frac{\zeta \times|E(\mathcal{H})|}{\gamma}=|E(\mathcal{G})| \times|A u t(\mathcal{H})|
$$

and thus

$$
\left|E\left(\mathcal{G}^{\prime}\right)\right| \geq \frac{\left|E\left(\mathcal{H}^{\prime}\right)\right| \times \zeta}{\gamma}=\frac{|E(\mathcal{G})| \times|A u t(\mathcal{H})|}{|E(\mathcal{H})|} \times\left|E\left(\mathcal{H}^{\prime}\right)\right| .
$$

Accordingly,

$$
\begin{aligned}
\bar{e}\left(\mathcal{G}^{\prime}\right) & =\frac{\left|E\left(\mathcal{G}^{\prime}\right)\right|}{\left|V\left(\mathcal{G}^{\prime}\right)\right|} \\
& \geq \frac{|E(\mathcal{G})| \times|A u t(\mathcal{H})| \times\left|E\left(\mathcal{H}^{\prime}\right)\right|}{|E(\mathcal{H})|} \times \frac{|V(\mathcal{H})|}{|V(\mathcal{G})| \times|A u t(\mathcal{H})| \times\left|V\left(\mathcal{H}^{\prime}\right)\right|} \\
& =\frac{|E(\mathcal{G})|}{|V(\mathcal{G})|} \times \frac{|V(\mathcal{H})|}{|E(\mathcal{H})|} \times \frac{\left|E\left(\mathcal{H}^{\prime}\right)\right|}{\left|V\left(\mathcal{H}^{\prime}\right)\right|} \\
& =\bar{e}(\mathcal{G}) \times \frac{1}{\bar{e}(\mathcal{H})} \times \bar{e}\left(\mathcal{H}^{\prime}\right) \\
& =\frac{\bar{e}(\mathcal{G})}{\bar{e}(\mathcal{H})} \times \xi(\mathcal{H}) .
\end{aligned}
$$

Consequently,

$$
\frac{\bar{e}(\mathcal{G})}{\bar{e}(\mathcal{H})} \times \xi(\mathcal{H}) \leq \bar{e}\left(\mathcal{G}^{\prime}\right) \leq \xi\left(\mathcal{G}^{\prime}\right)
$$


which yields

$$
\frac{\bar{e}(\mathcal{G})}{\bar{e}(\mathcal{H})} \leq \frac{\xi\left(\mathcal{G}^{\prime}\right)}{\xi(\mathcal{H})}
$$

One can simply check that $\xi\left(\mathcal{G}^{\prime}\right)=\xi(\mathcal{G})$, which finishes the proof.

By use of Theorem [2.6, we obtain the following corollary.

Corollary 2.7. Let $\mathcal{G}$ be a hypergraph. We have, $\chi_{f}(\mathcal{G}) \geq\left(\frac{|E(\mathcal{G})|}{|V(\mathcal{G})| \xi(\mathcal{H})}\right)+1$.

Proof. If $\chi_{f}(\mathcal{G})=\frac{m}{n}$, then there is a homomorphism from $\mathcal{G}$ to $\mathrm{KG}(t m, t n)$, for some positive integer $t$. Let $K_{0}$ be the induced subgraph of $\mathrm{KG}(t m, t n)$ by the vertices in $A \cup B$, where

$$
\begin{aligned}
& A=\{X \subseteq[t m]|| X \mid=t n, 1 \in X, 2 \notin X\}, \\
& B=\{X \subseteq[t m]|| X \mid=t n, 2 \in X, 1 \notin X\} .
\end{aligned}
$$

Clearly $K_{0}$ is a bipartite subgraph. In view of the definition of density, we have $\frac{\left|E\left(K_{0}\right)\right|}{\left|V\left(K_{0}\right)\right|} \leq$ $\xi(\operatorname{KG}(t m, t n))$. Consequently, using Theorem [2.6], we have

$$
\frac{\left|E\left(K_{0}\right)\right|}{\left|V\left(K_{0}\right)\right|} \times \frac{|V(\mathrm{KG}(t m, t n))|}{|E(\mathrm{KG}(t m, t n))|}=\frac{\left(\begin{array}{c}
t m-t n-1 \\
t n-1
\end{array}\right)}{\left(\begin{array}{c}
t m-t n \\
t n
\end{array}\right)}=\frac{1}{\chi_{f}(\mathcal{G})-1} \leq \frac{|V(\mathcal{G})| \xi(\mathcal{G})}{|E(\mathcal{G})|}
$$

as desired.

\section{Concluding remark}

In view of the technique used in Theorems [2.] and [2.6, we can prove some other similar results. Since the proofs are almost the same as the ones of these theorems, we bring these results here without proof.

Let $\mathcal{G}$ and $\mathcal{K}$ be two hypergraphs, we define

$$
\zeta(\mathcal{G}, \mathcal{K})=\max _{\mathcal{H} \leq \mathcal{G}}\left\{\frac{|E(\mathcal{H})|}{|V(\mathcal{H})|}: \mathcal{H} \longrightarrow \mathcal{K}\right\}
$$

By the following theorem, we generalize a result by Daneshgar and Hajiabolhassan [i] to the hypergraph case.

Theorem 3.1. Let $\mathcal{G}$ and $\mathcal{H}$ be two hypergraphs and $\mathcal{H}$ be a vertex and edge transitive hypergraph. If $f: \mathcal{G} \longrightarrow \mathcal{H}$ is a homomorphism, then

$$
\frac{\zeta(\mathcal{H}, \mathcal{K})}{\bar{e}(\mathcal{H})} \leq \frac{\zeta(\mathcal{G}, \mathcal{K})}{\bar{e}(\mathcal{G})}
$$

Similar as Corollary [2.7, we have the next corollary.

Corollary 3.2. Let $\mathcal{H}$ be a hypergraph, we have, $\chi_{f}(\mathcal{H}) \geq\left(\frac{|E(\mathcal{H})|}{|V(\mathcal{H})| \zeta\left(\mathcal{H}, K_{2}\right)}\right)+1$. 
Let $m$ be a fixed number. Define

$$
\sum_{m}(\mathcal{G})=\min \left\{\sum_{c}(\mathcal{G}) \mid c: \mathcal{G} \longrightarrow \mathrm{KG}^{r}(n, m) \text { is a homomorphism }\right\}
$$

where $\sum_{c}(\mathcal{G})=\sum_{v \in \mathcal{G}} \sum_{i \in c(v)} i$. Setting $m=1$ and $r=2$, next theorem implies a generalization of result by Alishahi and Taherkhani [2] to the hypergraph case.

Theorem 3.3. Let $\mathcal{G}$ and $\mathcal{H}$ be two hypergraphs. If $f: \mathcal{G} \longrightarrow \mathcal{H}$ is a homomorphism and $\mathcal{H}$ is a vertex transitive hypergraph, then we have

$$
\frac{\sum_{m}(\mathcal{G})}{|V(\mathcal{G})|} \leq \frac{\sum_{m}(\mathcal{H})}{|V(\mathcal{H})|}
$$

\section{ACKNOWLEDGMents}

The authors would like to acknowledge professor Meysam Alishahi for his valuable comments. They are also grateful to the referees for their careful reading and for their comments which helped us to improve the paper.

\section{REFERENCES}

[1] M. Alishahi and S. Tahmasebi, On circular chromatic number and chromatic number of some generalized Kneser hypergraph, To appear, Ars Combinatoria.

[2] M. Alishahi and A. Taherkhani, A note on chromatic sum, Ars Combinatoria. Vol. CXVI (2014), pp. 49-54.

[3] M. Alishahi and H. Hajiabolhassan, Circular coloring and Mycielski construction, Discrete mathematics. vol. 310, pp.1544-1550.

[4] J.A. Bondy and P. Hell, A note on the star chromatic number, Graph Theory. vol. 14 (1990),pp. 479-482.

[5] A.Daneshgar and H.Hajiabolhassan, Graph homomorphisms through random walks, J. Graph Theory. vol. 44 (2003),pp. 15-38.

[6] A. Daneshgar and H. Hajiabolhassan, Graph homomorphisms and nodal domains, Linear Algebra Appl. vol. 418 (2006),pp. $44-52$.

[7] A. Daneshgar and H. Hajiabolhassan, Density and power graphs in graph homomorphism problem, Discrete Mathematics. vol. 308 (2008), pp. 4027-4030.

[8] G. Hahn and C. Tardif, Graph homomorphisms: Structure and symmetry, in:G. Hahn,G.Sabidussi(Eds.) Graph Symmetry, in:, NATO Adv. Sci. Inst. Sci. Inst. Ser. C Math. Phys.Sci., no. 497, Kluwer, Dordrecht. (1997),pp. 107-167.

[9] P. Hell and J. Nesetril, Graph and homomorphisms, in:, Oxford Lecture Series in Mathematics and its Applications, vol. 28, Oxford University press, Oxford. (2004).

[10] H. Hajiabolhassan, On colorings of graph powers, Discrete Mathematics. vol. 310 (2009),pp. 4299-4305.

[11] H. Hajiabolhassan and A. Taherkhani, Graph powers and Graph homomorphism, Discrete Mathematics. vol. 310 (2010),pp. 4299-4305.

[12] S. Rahimi Sharbaf and Kh. Erfani, On the edge-difference and edge-sum chromatic sum of the simple graphs,Algebraic structures and their applications. vol. 4 (2017),pp. 33-42. 


\section{Samaneh Tahmasebi}

Faculty of Mathematical Sciences

Shahrood university of Technology, Shahrood

Shahrood, Iran.

samanehtahmasebi@shahroodut.ac.ir

\section{Sadegh Rahimi Sherbaf}

Faculty of Mathematical Sciences

Shahrood university of Technology, Shahrood

Shahrood, Iran.

srahimi@shahroodut.ac.ir 